\title{
Relative Frequency of Clostridium difficile in Patients with Diarrheal Disease
}

\author{
PETER H. GILLIGAN, $\dagger$ L. R. McCARTHY,* AND V. M. GENTA \\ Department of Hospital Laboratories, North Carolina Memorial Hospital, and Department of Bacteriology \\ and Immunology, University of North Carolina School of Medicine, Chapel Hill, North Carolina 27514
}

Received 31 July 1980/Accepted 23 March 1981

\begin{abstract}
We have studied 161 patients with diarrheal disease to determine the frequency with which Clostridium difficile occurs in such patients. $C$. difficile or its toxin or both were detected in stools from 19 patients (11.9\%), 17 of whom had previously received antimicrobial agents. Enteric pathogens other than $C$. difficile were recovered less frequently, with Salmonella sp., Giardia lamblia, and Campylobacter fetus being recovered from 4.1, 2.5, and 1.3\%, respectively, of the patients studied. These data suggest that $C$. difficile may be frequently encountered in specimens obtained from patients with diarrhea who receive antibiotics and may play a role in diarrheal disease in such patients.
\end{abstract}

Clostridium difficile has been implicated as an important etiological agent in antimicrobialassociated diarrheal disease and pseudomembranous colitis $(1,3,11)$. The pathogenicity of this organism is due to its ability to produce an extracellular protein that has cytotoxic effects on cells in tissue culture $(6,7)$ and can produce disease in experimental animals similar to that seen in humans $(2,10)$. Although the ability of C. difficile to produce pseudomembranous colitis has been well established $(1,3,11)$, the frequency of this organism in a general population with diarrheal disease has not been well established. In addition, the relative frequency of $C$. difficile in comparison to other enteric pathogens, such as Salmonella sp., Shigella sp., Yersinia sp., Campylobacter fetus, and intestinal parasites, has not been reported in the United States. In this paper, we present data that relate the frequency of isolation of $C$. difficile to that of other established enteric pathogens from patients with diarrheal disease.

\section{MATERIALS AND METHODS}

Patient population. The patients examined in this study were either inpatients or outpatients seen between March 1979 and April 1980 at the North Carolina Memorial Hospital, a tertiary care facility. All patients studied had watery diarrhea, which prompted a request for either bacterial stool culture or stool examination for ova and parasites. Watery diarrhea was defined as stools which took the form of the specimen container and could be easily pipetted with a pasteur pipette. Multiple specimens were often examined, with as many as seven specimens examined

† Present address: Clinical Microbiology Laboratory, St. Christopher's Hospital for Children, Philadelphia, PA 19133. per patient. Cultures for C. fetus, Clostridium difficile, Salmonella sp., Shigella sp., and Yersinia sp. were performed within $2 \mathrm{~h}$ of collection. Specimens for ova and parasites were also routinely examined for parasites within $2 \mathrm{~h}$ of collection. Those specimens submitted for parasitological examination that could not be examined within $2 \mathrm{~h}$ were mixed with $10 \%$ Formalin- $0.85 \%$ saline solution until examination. Stools that were to be assayed for $C$. difficile toxin were held at $-70^{\circ} \mathrm{C}$ until assay. All of the patients' charts were reviewed for diarrheal disease history and associated antimicrobial therapy.

Culture techniques. Of 77 patients whose specimens were cultured for $C$. fetus, 54 had their stools cultured on Skirrow medium (19). The plate medium was incubated in a candle jar at $37^{\circ} \mathrm{C}$ and examined daily for 3 days for colonies characteristic of $C$. fetus. Specimens from the remaining 23 patients were cultured on Campy-blood agar plates and in tubes of thioglycolate broth containing the same antibiotics found in Campy-BAP (4). The plate medium was incubated in a large GasPak jar (BBL Microbiology Systems, Cockeysville, Md.) by using two of the three catalyst baskets and two carbon dioxide-hydrogen gas generators. The plated medium was then processed, in the same manner previously described, for specimens obtained from the first 54 patients. The thioglycolate broth was incubated for 18 to $24 \mathrm{~h}$ in a water bath at $42^{\circ} \mathrm{C}$ and then subcultured to Campy-BAP and incubated as previously described. Colonies with morphology similar to that observed with Campylobacter colonies were examined by wet mount and, when necessary, further examined by Gram stain. Isolates which showed darting motility and a Gram stain similar to $C$. fetus were identified by using established techniques for $C$. fetus (20).

Stool specimens were examined for $C$. difficile by inoculating portions of each specimen onto a cycloserine-cefoxitin-fructose agar plate (12) which was then incubated in a GasPak jar for $48 \mathrm{~h}$. Colonies ferment- 
ing fructose, not degrading lecithin, and exhibiting a spreading margin were Gram stained and, when appropriate, identified by the anaerobe identification scheme of Holdeman et al. (14).

Stool examinations for ova and parasites were done by direct mounts in saline and Lugol iodine and, on occasion, by use of the trichrome stain (17). Stools were also concentrated by using Formalin-ether concentration techniques (18) or a modified concentration technique in which ethyl acetate was substituted for ether (21). Stool concentrates were suspended in saline and Lugol iodine and examined microscopically.

Salmonella and Shigella spp. were cultured by inoculating 5\% sheep blood, MacConkey, and Hektoen agar plates (BBL) and a GN enrichment broth with each specimen. The GN enrichment broth was subcultured after 6 to $18 \mathrm{~h}$ incubation at $37^{\circ} \mathrm{C}$ onto a Hektoen and MacConkey agar plate. The blood agar plate was used to detect overgrowth of the bowel flora with gram-positive organisms or yeasts. The MacConkey and Hektoen plates were examined for nonlactose-fermenting or hydrogen sulfide-producing organisms. Three colonies of each suspicious colony type were screened by using a triple sugar iron agar, a lysine iron agar, and a phenylalanine agar slant (8). Organisms with biochemical reactions consistent with those of either Salmonella or Shigella species were identified to serogroup by using standard biochemical and serological tests (8).

Yersinia enterocolitica was cultured by a cold enrichment method. After primary culture onto the media described above for Salmonella and Shigella spp., a stool swab was placed in $1.0 \mathrm{ml}$ of phosphatebuffered saline and incubated for 3 weeks at $4^{\circ} \mathrm{C}$. Subcultures were made weekly onto MacConkey and salmonella-shigella agar, and these plates were incubated at room temperature for $72 \mathrm{~h}$. These culture plates were examined daily for non-lactose-fermenting colonies similar to $Y$. enterocolitica. These isolates were characterized biochemically by using triple sugar iron agar or phenylalanine agar and two tubes of motility-indole-ornithine medium; one tube was incubated at room temperature and the other was incubated at $37^{\circ} \mathrm{C}$. Organisms with biochemical reactions consistent with those of $Y$. enterocolitica were identified by using appropriate biochemical tests (13). All dehydrated media used during the course of this study were supplied by either Difco Laboratories, Detroit, Mich., or BBL, unless otherwise noted.

C. difficile toxin assay. Frozen stool specimens were thawed in a water bath at $37^{\circ} \mathrm{C}$. A stool sample (ca. $0.5 \mathrm{ml}$ ) was placed in a plastic snap-cap tube (12 by $75 \mathrm{~mm}$ ) and diluted with an equal volume of phosphate-buffered saline containing $500 \mathrm{mg}$ of cycloserine. This mixture was mixed thoroughly and then centrifuged at $2,500 \times g$ for $10 \mathrm{~min}$. The stool supernatant was removed and used for the toxin assay. Those supernatants that either remained cloudy or contained particulate matter after centrifugation were filtered through a 0.45- $\mu \mathrm{m}$ membrane filter (Acrodisc; Gelman Sciences, Inc., Ann Arbor, Mich.) before assay. Either MRC-5 or WI-38 cells lines were grown in minimal essential medium supplemented with $10 \%$ fetal calf serum and $30 \mu \mathrm{g}$ of gentamicin per $\mathrm{ml}$. The cell mono- layers were prepared by placement of $0.2 \mathrm{ml}$ of cell suspension (at ca. $10^{5} / \mathrm{ml}$ ) into each well of a 96 -well flat-bottomed microtiter tissue culture plate (Costar, Cambridge, Mass.) and incubation at $37^{\circ} \mathrm{C}$ in $5 \% \mathrm{CO}_{2}$ until a monolayer formed. The assay for $C$. difficile toxin was performed by placing $50 \mu \mathrm{l}$ of $1: 2$ and $1: 10$ dilutions of each stool supernatant onto duplicate monolayers of one of the two fibroblast cell monolayers.

The diluted stool supernatant fluids were incubated with the cell monolayers at $37^{\circ} \mathrm{C}$ in $5 \% \mathrm{CO}_{2}$ for $48 \mathrm{~h}$. The monolayers were examined at 12 -h intervals during the incubation period. Those monolayers showing no morphological change after $48 \mathrm{~h}$ were considered negative. Those monolayers showing actinomorphic changes (2) in $>50 \%$ of the cell monolayer treated with the diluted stool supernatant were considered positive, and a neutralization assay was performed on those supernatants. Monolayers showing toxic changes such as cell lysis or cell fusion were also tested in the neutralization assay. Stool supernatants which caused contamination of the monolayers by microorganisms were filter sterilized as described above and again assayed.

Culture supernatant from a known toxigenic strain of $C$. difficile grown anaerobically in prereduced chopped meat medium (Scott Laboratories, Fiskeville, R.I.) at $37^{\circ} \mathrm{C}$ for $48 \mathrm{~h}$ was used as the positive standard for this assay. This toxigenic strain was kindly provided by V. R. Dowell of the Centers for Disease Control. Atlanta, Ga.

Neutralization assay. Confirmation of the presence of $C$. difficile toxin in patient specimens required the neutralization of cytotoxic activity with specific antibody. Supernatants showing actinomorphic or toxic changes at a 1:10 dilution were diluted in phosphate-buffered saline-cycloserine to give a final dilution of 1:10. The diluted supernatants were then mixed with a 1:10 dilution of antiserum to $C$. difficile toxin or a 1:10 dilution of fetal calf serum. The $C$. difficile antitoxin (9) used in this study was kindly supplied by Tracy Wilkins of the Virginia Polytechnic Institute and State University. This mixture was incubated at room temperature for $30 \mathrm{~min}$, and $100 \mu \mathrm{l}$ of the mixture was assayed as described above. Specimens causing cultured cells to demonstrate rounding in the presence of fetal calf serum and no rounding in the presence of C. difficile antitoxin were considered positive for the toxin.

Determination of toxin titers. Toxin titers were determined for each stool and culture supernatant fluid which was proven to be positive by the neutralization assay. Serial 10 -fold dilutions of the toxin sample from $10^{-1}$ to $10^{-6}$ were prepared in phosphatebuffered saline-cycloserine, and $50 \mu \mathrm{l}$ of each dilution was inoculated onto duplicate monolayers of either MRC-5 or WI-38 fibroblasts. The cells were examined for $48 \mathrm{~h}$ at 12 -h intervals. The last dilution to give greater than $50 \%$ rounding at $48 \mathrm{~h}$ was the toxin titer. When the culture supernatant from the control organism was used, the two cell lines proved to be equally susceptible to the toxin. 


\section{RESULTS}

Patient population. Of the 161 patients studied, 91 (57\%) developed diarrheal disease outside the hospital. Eighty-six patients (54\%) were male, and 124 patients $(76 \%)$ were over 25 years of age. A total of 129 patients $(80 \%)$ had diarrheal disease of less than 4 weeks in duration; 61 of these patients $(47 \%)$ had diarrhea associated with antimicrobial therapy, whereas $68(53 \%)$ had diarrhea that was not associated with antimicrobial therapy. There were 32 patients studied who had diarrheal disease of greater than 4 weeks in duration; 16 of these patients had chronic inflammatory bowel disease, and 16 had chronic diarrhea of unknown etiology.

Culture results. Specimens from the 161 patients were examined for the presence of $C$. difficile or its toxin. Specimens from 77, 113, 145 , and 85 patients were examined for $C$. fetus, intestinal parasites, Salmonella and Shigella spp., and Yersinia sp., respectively. Specimens from 139 patients were examined for three or more of the five groups of organisms. As can be seen in Table 1, C. difficile or its toxin was encountered in $12 \%$ of the patients. Salmonella sp. and Giardia lamblia were the only other organisms which were observed to occur in more than one patient. Y. enterocolitica and $C$. fetus subsp. jejuni were isolated from one patient each. Shigella sp. was not detected in any patient specimen. No patient studied had more than one enteric pathogen detected in his or her stools. Five additional patients who received prior antibiotic therapy had stool cultures that revealed an absence of Enterobacteriaceae and a predominance of Pseudomonas aeruginosa or yeasts.

Detection of $C$. difficile or its toxin. Of the 161 patients studied, specimens of 110 were examined for both $C$. difficile and its toxin; specimens of 51 were tested for the presence of toxin only. The results of culture and toxin assays performed on the 19 patients whose specimens

TABLE 1. Recovery of enteric pathogens from patients with diarrheal disease

\begin{tabular}{lcc}
\hline \multicolumn{1}{c}{ Organism } & $\begin{array}{c}\text { No. of pa- } \\
\text { tients } \\
\text { studied }\end{array}$ & $\begin{array}{c}\text { No. (\%) of pa- } \\
\text { tients with posi- } \\
\text { tive examina- } \\
\text { tions }\end{array}$ \\
\hline C. fetus & 77 & $1(1.3)$ \\
C. difficile & 161 & $19(10.4)$ \\
Intestinal parasites ${ }^{a}$ & 113 & $4(3.4)$ \\
Salmonella sp. & 145 & $6(4.1)$ \\
Shigella sp. & 145 & $0(0)$ \\
Yersinia sp. & 82 & $1(1.2)$ \\
\hline
\end{tabular}

${ }^{a}$ One Ascaris lumbricoides, three Giardia lamblia. yielded C. difficile or its toxin were as follows. In 13 patients, both the toxin and the organism were present, in 4 , only the toxin was detected, and in 2 , only the organism was detected. Of the four patients in whom only $C$. difficile toxin was detected, two were not cultured for the organism and two were culture negative. One of the two patients who was toxin positive and culture negative had been treated with oral vancomycin for $24 \mathrm{~h}$ before culture. $C$. difficile-associated diarrhea in these four patients was diagnosed by detecting $C$. difficile toxin in their stool specimen(s). The relative concentration of $C$. difficile toxin was determined for stool specimens obtained from these 4 patients and for the 13 patients whose specimens were positive for both toxin and culture. These 17 specimens were positive for toxin at dilutions ranging from $10^{-1}$ to $10^{-5}$. Specimens from 11 of 17 patients yielded positive toxin assays with dilutions $\geq 2 \times 10^{-2}$. No difference was observed in the levels of toxin present in stool specimens that were culture positive when compared with those that were culture negative.

Two patients had $C$. difficile detected in their specimens by culture only. Assay for toxin on these two stools was negative on three separate occasions. Both strains were tested to determine if they produced toxin in vitro. One of the two strains produced a cytotoxin when it was grown anaerobically in prereduced $1 \%$ glucose-chopped meat broth. The toxin titer produced by this organism in culture was $5 \times 10^{-2}$. The patient from whom this strain was recovered had mild diarrheal disease which resolved without therapy. The other strain that was recovered from a specimen that did not contain free toxin failed to produce the toxin when grown anaerobically. This latter strain did not produce toxin when grown in chopped meat-1\% glucose, Schadler broth, thioglycolate broth, and Trypticase soy broth (BBL Microbiology Systems). The patient suffered from several daily episodes of severe diarrhea which resolved after empiric treatment with oral vancomycin. $C$. difficile could not be recovered from cultures of stool specimens obtained after completion of 5 days of vancomycin therapy.

Association of $C$. difficile with antibiotic therapy. Of the 161 patients studied, 61 received antimicrobial therapy before culture. Of these patients, $17(28 \%)$ had $C$. difficile or its toxin present in their feces. No established enteric pathogen was found in specimens obtained from these 61 patients.

Prior antibiotic therapy was not associated with the remaining 100 patients studied, and $C$. difficile was recovered from only two of these 
patients. One of these patients was a 2-year-old female (positive $C$. difficile culture; stool toxin concentration of $2 \times 10^{-1}$ ) diagnosed as having viral gastroenteritis and whose symptoms resolved without antimicrobial therapy. The other patient was a 69-year-old male (stool toxin concentration of $1 \times 10^{-2}$ ) who had diarrhea that resolved with supportive therapy.

Antimicrobial agents most frequently administered to patients with diarrhea that was associated with positive culture or toxin assays for C. difficile were penicillins, cephalosporins, and aminoglycosides. Penicillins were administered in 12 of 17 patients who had toxin assays or cultures positive for $C$. difficile. Cephalosporins and aminoglycosides were each given to $11 \mathrm{pa}$ tients. Prior therapy with clindamycin, chloramphenicol, tetracycline, or trimethroprim-sulfamethoxazole was also associated with two or more patients that developed diarrhea and whose specimens were toxin- or culture-positive for $C$. difficile. Of these 17 patients, 11 received simultaneous therapy with two or more antimicrobial agents, one always being an aminoglycoside. Six additional patients were treated with only one antibiotic which was either a penicillin or cephalosporin. The time of onset of disease after initiation of antimicrobial therapy ranged from 2 to 23 days with an average of 13 days.

The patients who had antimicrobial agent-associated diarrhea with a positive $C$. difficile culture or toxin assay were older than 25 years. Only 5 of 12 patients who had disease caused by other enteric pathogens were older than 25. Three of these latter five patients had G. lamblia infections. Neither $C$. difficile nor any other enteric pathogen was found to be associated with diarrheal disease of greater than four weeks duration. All cases of diarrhea caused by established enteric pathogens were outpatients. The two patients who had C. difficile-associated disease but did not have a history of prior antimicrobial therapy also developed disease outside the hospital. Of the 17 patients with antimicrobial associated diarrhea with evidence of $C$. difficile, 12 developed symptoms during hospitalization, and five developed symptoms as outpatients. Three of the latter five developed symptoms before hospitalization, whereas the other two developed symptoms less than a month after release from the hospital.

Sigmoidoscopic examinations were performed on 70 of 161 patients studied (Table 2). The examination results were reported as either normal, nonspecific colitis (including inflammatory bowel disease), or pseudomembranous colitis. If the patients with inflammatory bowel disease were eliminated from the $C$. difficile-
TABLE 2. Sigmoidoscopic examination of patients with diarrheal disease

\begin{tabular}{|c|c|c|c|}
\hline \multirow[b]{2}{*}{ Patient type } & \multicolumn{3}{|c|}{ No. of patients diagnosed as: } \\
\hline & Normal & $\begin{array}{l}\text { Nonspecific } \\
\text { colitis }\end{array}$ & $\begin{array}{c}\text { Pseudo- } \\
\text { mem- } \\
\text { branous } \\
\text { colitis }\end{array}$ \\
\hline \multicolumn{4}{|l|}{ C. difficile positive ${ }^{a}$} \\
\hline Antibiotic treatment & 3 & 7 & 2 \\
\hline $\begin{array}{l}\text { No antibiotic treat- } \\
\text { ment }\end{array}$ & 0 & 1 & 0 \\
\hline \multicolumn{4}{|l|}{ C. difficile negative } \\
\hline Antibiotic treatment & 10 & 8 & 0 \\
\hline $\begin{array}{l}\text { No antibiotic treat- } \\
\text { ment }\end{array}$ & 13 & 11 & 0 \\
\hline $\begin{array}{l}\text { Inflammatory bowel } \\
\text { disease }\end{array}$ & 2 & 13 & 0 \\
\hline
\end{tabular}

${ }^{a}$ Individuals had toxin or organism or both present in their stool specimens.

negative patients, $C$. difficile-positive patients had a higher percentage of abnormal sigmoidoscopies $(77 \%)$ as compared with the $C$. difficilenegative patients $(48 \%)$. In addition, pseudomembranes were seen on sigmoidoscopy in two of the C. difficile-positive patients, but in none of the $C$. difficile-negative patients.

Since the disease of most patients who were C. difficile culture- or toxin-positive or both was resolved by using either supportive therapy (discontinuing antibiotics, using fluid therapy, and administration of Lomotil) or vancomycin therapy, few follow-up data on this group of patients were available. Almost all of the patients included in this study who had multiple specimens tested had neither $C$. difficile nor an established pathogen recovered. One patient who was positive at admission, by both culture and toxin assay for $C$. difficile, was closely studied for a period of 2 months. She was treated for 2 weeks with oral tetracycline, 3 weeks before admission. At admission, she had severe diarrheal disease. A sigmoidoscopic examination at that time revealed diffuse edema and local inflammation, but no pseudomembranes were seen. She was also culture- and toxin-positive for $C$. difficile. Oral vancomycin (500 $\mathrm{mg}$ twice a day) was administered for 5 days, and her diarrhea quickly resolved. Toxin and culture studies 2 weeks after cessation of therapy were both negative. She was readmitted 10 days later with severe diarrhea and, again, was culture- and toxin-positive with a 10-fold increase in her stool toxin titer. She was again treated with the same course of vancomycin previously given and her disease once again resolved. A week after the cessation of therapy she was again culture and toxin negative and did not return with any symptoms for the next 6 months. 


\section{DISCUSSION}

C. difficile has been implicated as an important etiological agent of antimicrobial-associated diarrhea and pseudomembranous colitis (1-3, 11). However, the relative frequency of this organism in patients with diarrheal disease in comparison to that of other enteric pathogens is not known. Our results indicate that $C$. difficile is an organism that may be commonly recovered from patients with diarrheal disease in our institution. A total of $90 \%$ of the $C$. difficile-positive patients were receiving or recently received antibiotic therapy; this supports the view that antibiotic therapy was an important predisposing factor for the establishment of $C$. difficile in the intestine $(1-3,11)$. Our finding that $100 \%$ of patients with pseudomembranous colitis and $27 \%$ of patients with antibiotic-associated diarrheal disease harbored $C$. difficile agreed with the finding of Bartlett et al. (3). In addition, we found that the concentrations of $C$. difficile toxin in the stools we examined were similar to those reported by Bartlett et al. (2). These investigators observed that the concentration of toxin varied widely in patients with $C$. difficile-associated pseudomembranous colitis, and they ascribed this finding to the differing number of organisms present in the stool specimens they tested. In our patient population, there appeared to be no correlation between toxin titer and severity of disease. However, patients whose specimens were positive for $C$. difficile had a higher percentage of abnormal sigmoidoscopic examinations when compared with patients whose stools were negative for $C$. difficile, suggesting a role for $C$. difficile in the pathogenesis of disease in our patient population. Further support for such a role in antibiotic-associated diarrheal disease was that therapy effective in pseudomembranous colitis caused by $C$. difficile was also effective in at least one patient with antibiotic-associated diarrhea with positive cultures and toxin assays. Many questions concerning the pathogenesis of antibiotic-associated diarrhea and the possible role of $C$. difficile in this disease remain unanswered.

Two or our patients had stool specimens which did not contain toxin but had cultures that yielded $C$. difficile. One of the patients had a toxigenic organism and mild diarrheal disease which resolved without treatment. The role that C. difficile may have played in this patient's diarrhea is difficult to assess. The other patient had severe diarrhea that resolved with oral vancomycin therapy and elimination of a nontoxigenic strain of $C$. difficile from stool specimens. The observation of disease associated with the presence of a nontoxigenic strain of $C$. difficile may be coincidental or may suggest that the $C$. difficile produces another toxin(s) which may play a role in diarrheal disease. Clostridia, such as C. perfringens, produce multiple toxins (16). Therefore, the production of multiple toxins by C. difficile would not be unusual.

It has recently been reported that $C$. difficile may play a role in relapse in chronic inflammatory bowel disease $(5,15)$. None of the 16 patients included in our study who had inflammatory bowel disease and were suffering relapse had stool specimens positive for $C$. difficile or its toxin.

The role for $C$. difficile as a cause of pseudomembranous colitis is well established $(1-3,11)$. Data presented here and by Bartlett et al. (3) suggest that $C$. difficile may play an important role in the pathogenesis of antibiotic-associated diarrhea. Because of the proven and possible importance of this organism in severe diarrheal disease, clinical laboratories in tertiary care facilities should consider establishing techniques for the detection of $C$. difficile and its toxin.

\section{ACKNOWLEDGMENT}

We thank Jean Bowdre for assistance given throughout the course of this study.

\section{LITERATURE CITED}

1. Bartlett, J. G., T. W. Chang, M. Gurwith, S. L. Gorbach, and A. B. Onderdonk. 1978. Antibiotic-associated pseudomembranous colitis due to toxin-producing clostridia. N. Engl. J. Med. 298:531-534.

2. Bartlett, J. G., T. W. Chang, N. S. Taylor, and A. B. Onderdonk. 1979. Colitis induced by Clostridium difficile. Rev. Infect. Dis. 1:370-378.

3. Bartlett, J. G., N. S. Taylor, T. W. Chang, and J. Dzink. 1980. Clinical and laboratory observations in Clostridium difficile colitis. Am. J. Clin. Nutr. 33:25212528.

4. Blaser, M. J., J. Cravens, B. W. Powers, F. M. LaForce, and W. L. Wang. 1979. Campylobacter enteritis associated with unpasteurized milk. Am. J. Med. 67:715-718.

5. Bolton, R. P., R. J. Sherriff, and A. E. Read. 1980. Clostridium difficile associated diarrhea: a role in inflammatory bowel disease? Lancet (ii): 383-384.

6. Chang, T. W., P. S. Lin, S. L. Gorbach, and J. G. Bartlett. 1979. Ultrastructural changes of cultured human amnion cells by Clostridium difficile toxin. Infect. Immun. 23:995-998.

7. Donta, S. T., and S. J. Shaffer. 1980. Effects of Clostridium difficile toxin on tissue-cultured cells. J. Infect. Dis. 141:218-222.

8. Edwards, P. R., and W. H. Ewing. 1972. Identification of Enterobacteriaceae. Burgess Publishing Co., Minneapolis.

9. Ehrich, M., R. L. Van Tassell, J. M. Libby, and T. D. Wilkins. 1980. Production of Clostridium difficile antitoxin. Infect. Immun. 28:1041-1043.

10. Fekety, R., J. Silva, R. Toshniwal, M. Allo, J. Armstrong, R. Browne, J. Ebright, and G. Rifkin. 1979. Antibiotic-associated colitis: effects of antibiotics on Clostridium difficile and the disease in hamsters. Rev. Infect. Dis. 1:386-397. 
11. George, W. L., R. D. Rolfe, V. L. Sutter, and S. M. Finegold. 1979. Diarrhea and colitis associated with antimicrobial therapy in man and animals. Am. J. Clin. Nutr. 32:251-257.

12. George, W. L., V. L. Sutter, D. Citron, and S. M. Finegold. 1979. Selective and differential medium for isolation of Clostridium difficile. J. Clin. Microbiol. 9: 214-219.

13. Hawkins, T. H., and D. J. Brenner. 1978. Isolation and identification of Yersinia enterocolitica. Centers for Disease Control, Atlanta.

14. Holdeman, L. V., E. P. Cato, and W. E. C. Moore (ed.). 1977. Anaerobe laboratory manual, 4th ed. Virginia Polytechnic Institute, Blacksburg.

15. LaMont, J. T., and Y. M. Trnka. 1980. Therapeutic implications of Clostridium difficile toxin during relapse of chronic inflammatory bowel disease. Lancet i: 381-383.
16. MacLennan, J. D. 1962. The histotoxic clostridial infections of man. Bacteriol. Rev. 26:177-276.

17. Melvin, D. M., and M. M. Brooke. 1974. Laboratory procedures for the diagnosis of intestinal parasites. Centers for Disease Control publication no. 75-8282. U.S. Department of Health, Education, and Welfare, Washington, D.C.

18. Ritchie, L. S. 1948. An ether sedimentation technique for routine stool examination. Bull. U.S. Army Med. Dep. 8:326.

19. Skirrow, M. B. 1977. Campylobacter enteritis: a new disease. Brit. Med. J. 2:9-11.

20. Smibert, R. M. 1978. The genus Campylobacter. Annu. Rev. Microbiol. 32:673-709.

21. Young, K. H., S. L. Bullock, D. M. Melvin, and C. L. Spruill. 1979. Ethyl acetate as a substitute for diethyl ether in the formalin-ether sedimentation technique. J. Clin. Microbiol. 10:852-853. 